\title{
Do community demographics, environmental characteristics and access to care affect risks of developing ACOS and mortality in people with asthma?
}

\author{
To the Editor:
}

Individuals with asthma and chronic obstructive pulmonary disease (COPD) overlap syndrome (ACOS) have a more rapid decline in lung function, more frequent exacerbations and worse quality of life than those with asthma or COPD alone [1-3]. Various risk factors may be associated with the development of ACOS, such as smoking history and status, obesity, comorbidity and indoor and outdoor environmental exposures $[1,4-6]$. The risk of developing ACOS may vary substantially by region, since demographic and environmental risk factors and community characteristics are not geographically homogeneous. Here, we use population-based data to estimate the incidence of ACOS in the asthma population and to measure the association between demographic factors, community-level characteristics and environmental factors and the risk of incident ACOS and all-cause mortality while accounting for spatial autocorrelation.

A cohort approach was used to follow individuals with incident asthma in Ontario, Canada aged $\geqslant 18$ years in 1996 , to determine the incidence of COPD (i.e. ACOS). While individuals with ACOS may consist of various phenotypes $[1,7,8]$, in this paper, our focus is on those with physician-diagnosed asthma who subsequently received a physician diagnosis of COPD. Using validated asthma and COPD health administrative case definitions $[9,10]$ and linking individual level data across multiple provincial health administrative databases (hospital discharges, emergency department visits, physician claims and death certificates), we identified incident asthma in 1996-2009, incident COPD in 1998-2014 and all-cause mortality in 1996-2014. In order to examine geographical variations in outcomes and risk factors, we applied an ecological design using data at the Census Division (CD) level. In Ontario, there are $49 \mathrm{CDs}$ that vary in size $\left(663-439000 \mathrm{~km}^{2}\right)$ and population density $\left(0.1-4150\right.$ people per $\left.\mathrm{km}^{2}\right)$.

To distinguish between incident and prevalent cases, a minimum 5-year asthma- or COPD-free observation period prior to the incidence date was applied. Those without a valid health card number for data linkage, missing residence postal code, age at COPD diagnosis $<35$ years or with COPD diagnosis prior to asthma were excluded. To minimise potential false positives (i.e. an initial asthma diagnosis that was later changed to COPD), those with COPD diagnosed $\leqslant 2$ years after asthma incidence were excluded ( $\mathrm{n}=26591,6 \%$ of asthma population).

The following risk factors were calculated at the $\mathrm{CD}$ level: 1) the Ontario Marginalization Index (ON-Marg), a proxy measure of socioeconomic status (SES) measured using four dimensions: material deprivation, residential instability, ethnic concentration and dependency [11];2) smoking prevalence rates derived from the 2013 Canadian Community Health Survey; 3) the Air Quality Health Index (AQHI), a composite air pollution index based on levels of ozone $\left(\mathrm{O}_{3}\right)$, fine particles with a diameter of $2.5 \mu \mathrm{m}$ or less (PM2.5) and nitrogen dioxide $\left(\mathrm{NO}_{2}\right)$ [12], measured from 49 fixed-site monitors around the province; and 4) density of and travel times calculated along a driving network to respiratory healthcare professionals (respiratory therapists and respirologists) and pulmonary function laboratories.

Poisson regression models were fitted to the data to measure the association between the risk factors described above and observed counts of incident ACOS and all-cause mortality. The log of the expected counts of the outcome, calculated based on provincial age- and sex-specific rates, were used as an offset. A

@ERSpublications

Material deprivation increases ACOS and death risk in people with asthma; air pollution may also increase death risk http://ow.ly/Eb8730dRPPc

Cite this article as: To T, Zhu J, Carlsten C, et al. Do community demographics, environmental characteristics and access to care affect risks of developing ACOS and mortality in people with asthma? Eur Respir J 2017; 50: 1700644 [https://doi.org/10.1183/13993003.00644-2017]. 
stochastic spatial random effect term was added to account for spatial autocorrelation. The $\mathrm{R}$ package CARBayes was used to calculate adjusted risk ratios (RR) and Bayesian 95\% credible regions (CR) [13].

Of the 414568 Ontarians with incident physician-diagnosed asthma included in the study, 32867 (7.9\%) were diagnosed with incident COPD, and 39022 (9.4\%) died. Among those who developed COPD, the median time from asthma incidence to COPD was 6.2 years (interquartile range (IQR) 3.8-9.4). Among those who died, the median time from asthma incidence to death was 7.8 years (IQR 4.9-11.1). The median yearly average AQHI was 3.3, corresponding to a low health risk. The median community-level prevalence of current smoking was $21.3 \%$. The density of respiratory therapists was relatively high (15.3 per 100000 population) compared to that of respirologists ( 0.74 per 100000 population) and pulmonary function laboratories ( 0.67 per 100000 population). Median travel times to respiratory healthcare professionals and pulmonary function laboratories were all under $30 \mathrm{~min}$.

Table 1 contains Bayesian risk estimates for the incidence of ACOS and mortality (adjusted for covariates and both unadjusted and adjusted for spatial autocorrelation).

After adjusting for covariates and spatial autocorrelation, those living in more materially deprived areas had a $46 \%$ higher risk of incident ACOS (RR 1.46, 95\% CR 1.13, 1.95) and a $28 \%$ higher risk of mortality (RR 1.28, 95\% CR 1.12, 1.46). Those living in areas with a higher ethnic concentration had a reduced risk of incident ACOS (RR 0.72, 95\% CR 0.58, 0.87) and mortality (RR 0.82, 95\% CR 0.75, 0.90). Our data suggested that increased AQHI was associated with an increased risk of incident ACOS (RR 1.44, 95\% CR $0.91,2.18$ ) and mortality (RR $1.24,95 \%$ CR 1.00, 1.50), but these associations were not significant. There were no significant associations between the outcomes and residential instability, dependency, community-level smoking prevalence or access to respiratory healthcare professionals or pulmonary function laboratories.

Our finding of material deprivation being associated with a higher risk of incident ACOS and mortality is consistent with findings of low SES being associated with higher respiratory diseases morbidity [14]. Despite universal healthcare, individuals may still have a financial barrier to chronic disease management (e.g. cost of prescription medications, which are not routinely covered in Ontario).

Areas with a higher ethnic concentration had a lower risk of mortality and incident ACOS. OMARIBA [15] found that Canadian immigrants have lower avoidable mortality than non-immigrants and suggested that differential access to and use of health services, differences in protective health-related behaviour, and the

TABLE 1 Unadjusted and adjusted Bayesian spatial model risk estimates and their respective 95\% credible regions (CRs)

Covariates
Incidence of ACOS

\begin{tabular}{|c|c|}
\hline $\begin{array}{l}\text { Unadjusted for } \\
\text { spatial } \\
\text { autocorrelation }\end{array}$ & $\begin{array}{l}\text { Adjusted for } \\
\text { spatial } \\
\text { autocorrelation }\end{array}$ \\
\hline $95 \% \mathrm{CR}$ & $95 \% \mathrm{CR}$ \\
\hline
\end{tabular}

All-cause mortality

\begin{tabular}{crrrr}
\hline \multicolumn{2}{c}{$\begin{array}{c}\text { Unadjusted for } \\
\text { spatial } \\
\text { autocorrelation }\end{array}$} & & & \multicolumn{2}{c}{$\begin{array}{c}\text { Adjusted for } \\
\text { spatial } \\
\text { autocorrelation }\end{array}$} \\
\hline RR & $95 \%$ CR & & RR & $95 \%$ CR
\end{tabular}

1.62

1.07

$(1.25,2.05)$

$(0.80,1.44)$

1.46

$(0.63,0.84)$

1.10
$\mathbf{0 . 7 2}$

$1.45 \quad(1.17,1.78)$

(1.02, 1.05)

0.88

1.00

$(0.37,1.40)$

1.44

(1.13, 1.95)

$(0.80,1.52)$

$(0.58,0.87)$

$(0.69,1.11)$

$(0.98,1.02)$

$(0.91,2.18)$

$(0.77,1.08)$

0.94

1.04

1.08

$(0.93,1.19)$

1.00

$(0.96,1.23)$

1.04

0.98

$(0.89,1.13)$

$(0.96,1.12)$

$(0.89,1.08)$

1.02

1.05

1.00

$(0.98,1.10)$

$(0.99,1.01)$

$(0.89,1.00)$

1.00

(0.97, 1.08)

$(0.99,1.01)$

$(0.92,1.00)$

0.02

0.40

472.45
$(0.01,0.06)$

$(0.03,0.89)$

$\mathbf{1 . 3 4}$
1.15
$\mathbf{0 . 8 7}$
$\mathbf{1 . 2 3}$
$\mathbf{1 . 0 2}$
0.88

1.00
1.02
1.01

1.01
1.00
0.98

1.34

$(1.18,1.50)$

1.28

$(0.97,1.40)$

$(0.81,0.95)$

$(1.08,1.37)$

$(1.01,1.03)$

$(0.62,1.24)$

1.09

0.82

0.92

1.00

1.24

$(0.92,1.10)$

0.99

$(0.94,1.10)$

1.01

$(0.94,1.09)$

0.99

$(1.12,1.46)$

$(0.94,1.28)$

$(0.75,0.90)$

$(0.79,1.07)$

$(0.99,1.01)$

$(1.00,1.50)$

$(0.93,1.05)$

(0.97, 1.07)

(0.97, 1.05)

0.99

$(0.99,1.01)$

1.00

$(0.95,1.02)$

1.00

0.00

0.52

460.60
$(0.95,1.04]$

$(0.96,1.02)$

$(0.99,1.00)$

$(0.98,1.02)$

$(0.00,0.02)$

$(0.06,0.93)$

Bold values represent risk estimates and $95 \%$ credible regions that do not include 1.00 . 
"healthy immigrant effect" may play a role. Similarly, lower rates of ACOS in areas with higher ethnic concentration may be attributable to the healthy immigrant effect or underuse of healthcare services.

Some limitations of this study should be noted. Individual-level data on comorbidity, lifestyle factors, health-seeking behaviour, availability of a drug plan and adherence to prescribed medication were not available, which limits our ability to draw definitive conclusions concerning factors that drive individual-level risks of incident ACOS and mortality. Community-level smoking was used as a proxy to measure tobacco exposure, which may obscure the effect of individual smoking on the outcomes of interest. Access to smoking cessation programmes and Certified Respiratory Educators (who may be respiratory therapists, nurses or pharmacists) trained to provide chronic disease management are important unmeasured access to care factors.

In summary, community-level smoking prevalence and access to respiratory healthcare professionals and pulmonary function labs had little, if any, effect on incident ACOS and all-cause mortality in the asthma population. Our results suggested that air pollution may be associated with an increased risk of all-cause mortality. Material deprivation was the only risk factor that was associated with both increased incident ACOS and all-cause mortality, suggesting that factors outside of the universal healthcare system may play an important role in health outcomes of marginalised populations. Focusing on barriers faced by marginalised population, such as improving sub-standard housing conditions and increasing awareness of health effects of air pollution, may be more effective strategies to improving health outcomes for asthma patients at risk of ACOS and mortality.

Teresa To ${ }^{1,2,3}$, Jingqin $\mathrm{Zhu}^{1,2}$, Christopher Carlsten ${ }^{4}$, Kristian Larsen ${ }^{1,5}$, Kandace Ryckman ${ }^{1}$, Laura Y. Feldman ${ }^{1}$, Eric Crighton, ${ }^{2,6}$ M. Diane Lougheed ${ }^{2,7}$, Christopher Licskai ${ }^{8}$, Paul J. Villeneuve ${ }^{3,9}$, Yushan Su ${ }^{10}$, Mohsen Sadatsafavi ${ }^{11}$ and Andrea Gershon ${ }^{1,2,3,12}$, for the Canadian Respiratory Research Network

${ }^{1}$ Child Health Evaluative Sciences, The Hospital for Sick Children, Toronto, ON, Canada. ${ }^{2}$ Institute for Clinical Evaluative Sciences, Toronto, ON, Canada. ${ }^{3}$ Dalla Lana School of Public Health, Toronto, ON, Canada. ${ }^{4}$ Institute for Heart and Lung Health, University of British Columbia, Vancouver, BC, Canada. ${ }^{5}$ Dept of Geography, University of Toronto Mississauga, Mississauga, ON, Canada. ${ }^{6}$ Dept of Geography, Environment and Geomatics, University of Ottawa, Ottawa, ON, Canada. ${ }^{7}$ Dept of Medicine, Queen's University, Kingston, ON, Canada. ${ }^{8}$ Schulich School of Medicine and Dentistry, Western University, London, ON, Canada. ${ }^{9}$ Dept of Health Sciences, Carleton University, Ottawa, ON, Canada. ${ }^{10}$ Ontario Ministry of the Environment and Climate Change, Toronto, ON, Canada. ${ }^{11}$ Faculty of Pharmaceutical Sciences and Dept of Medicine, University of British Columbia, Vancouver, BC, Canada. ${ }^{12}$ Sunnybrook Health Sciences Centre, Toronto, ON, Canada.

Correspondence: Teresa To, Hospital for Sick Children, Child Health Evaluative Sciences, 686 Bay Street, Toronto, Ontario M5G 0A4, Canada. E-mail: teresa.to@sickkids.ca

Received: Feb 282017 | Accepted after revision: June 122017

Support statement: This study was funded by the Canadian Respiratory Research Network (CRRN). The CRRN is supported by grants from the Canadian Institutes of Health Research (CIHR)-Institute of Circulatory and Respiratory Health; Canadian Lung Association (CLA)/Canadian Thoracic Society (CTS); British Columbia Lung Association; and Industry Partners Boehringer-Ingelheim Canada Ltd, AstraZeneca Canada Inc., and Novartis Canada Ltd. Funding for training of graduate students and new investigators within the network was supported by the above funding sponsors and as well by GlaxoSmithKline Inc. The funders had no role in the study design, data collection and analysis, or preparation of the manuscript. The opinions, results and conclusions presented in this report are those of the authors and are independent from the funding sources. Funding information for this article has been deposited with the Crossref Funder Registry.

Conflict of interest: Disclosures can be found alongside this article at erj.ersjournals.com

Acknowledgements: Health administrative data were provided by the Institute for Clinical Evaluative Sciences (ICES) and air pollution data were provided by the Ministry of the Environment and Climate Change (MOECC). Neither ICES nor the MOECC had any role in study design, analysis, interpretation of data, or writing of the report. No endorsement by ICES or the MOECC is intended or should be inferred. Practice locations of currently certified respirologists were obtained from the website of the College of Physicians and Surgeons of Ontario (www.cpso.on.ca/). Practice location of currently certified asthma/COPD educators and certified respiratory therapists were provided by the College of Respiratory Therapists of Ontario. Finally, the geographic locations of pulmonary function testing laboratories or spirometry clinics were obtained from the Canadian Lung Association (http://sct.poumon.ca/respDB/search-testing_e.php).

Kristian Larsen received a Postdoctoral Fellowship, in part, through the Hospital for Sick Children Research Training Centre and the CRRN. Andrea Gershon holds a New Investigator Career Award from CIHR and was also the recipient of 2015 Early Career Achievement Award of the Assembly on Behavioural Science and Health Services Research, American Thoracic Society. Teresa To was the recipient of the 2016 Meritorious Service Award of the Ontario Lung Association.

Author contributions are as follows. T. To initiated and designed the study, conducted the spatial modelling and statistical analysis, interpreted findings and drafted the manuscript. J. Zhu compiled the data and conducted statistical analysis. K. Larsen compiled the geographical variables and conducted GIS mapping. L.Y. Feldman and K. Ryckman conducted a search of the literature, summarised relevant study findings and reviewed the manuscript. All authors interpreted findings, reviewed and commented on drafts, have seen and approved the final version. 


\section{References}

1 Gibson PG, Simpson JL. The overlap syndrome of asthma and COPD: what are its features and how important is it? Thorax 2009; 64: 728-735.

2 Kauppi P, Kupiainen H, Lindqvist A, et al. Overlap syndrome of asthma and COPD predicts low quality of life. J Asthma 2011; 48: 279-285.

3 Rhee CK, Yoon $\mathrm{HK}$, Yoo KH, et al. Medical utilization and cost in patients with overlap syndrome of chronic obstructive pulmonary disease and asthma. COPD 2014; 11: 163-170.

4 Alshabanat A, Zafari Z, Albanyan O, et al. Asthma and COPD overlap syndrome (ACOS): a systematic review and meta analysis. PLoS One 2015; 10: e0136065.

5 Papaiwannou A, Zarogoulidis $\mathrm{P}$, Porpodis $\mathrm{K}$, et al. Asthma-chronic obstructive pulmonary disease overlap syndrome (ACOS): current literature review. J Thorac Dis 2014; 6: Suppl. 1, S146-S151.

6 Andersen ZJ, Bønnelykke K, Hvidberg M, et al. Long-term exposure to air pollution and asthma hospitalisations in older adults: a cohort study. Thorax 2012; 67: 6-11. Orie NG. The Dutch hypothesis. Chest 2000; 117: Suppl. 1, 299S.

Gibson PG, McDonald VM. Asthma-COPD overlap 2015: now we are six. Thorax 2015; 70: 683-691.

Gershon AS, Wang C, Guan J, et al. Identifying patients with physician diagnosed asthma in health administrative databases. Can Respir J 2009; 16: 183-188.

10 Gershon A, Wang C, Guan J, et al. Identifying individuals with physician diagnosed COPD in health administrative databases. COPD 2009; 6: 388-394

11 Matheson FI, Dunn JR, Smith KLW, et al. On-Marg Ontario Marginalization Index User Guide. Toronto, Centre for Research on Inner City Health, 2006.

12 Stieb DM, Burnett RT, Smith-Doiron M, et al. A new multipollutant, no-threshold air quality health index based on short-term associations observed in daily time-series analyses. J Air Waste Manag Assoc 2008; 58: 435-450.

13 Lee D. CARBayes: Spatial Generalised Linear Mixed Models for Areal Unit Data. 2016. Version 4.6: Vignette for CARBayes package. https:/CRAN.R-project.org/package=CARBayes Last accessed: September 2016.

14 Bacon SL, Bouchard A, Loucks EB, et al. Individual-level socioeconomic status is associated with worse asthma morbidity in patients with asthma. Respir Res 2009; 10: 125.

15 Omariba DW. Immigration, ethnicity, and avoidable mortality in Canada, 1991-2006. Ethn Health 2015; 20 409-436. 